\title{
Spinal cord involvement in a Burkitt lymphoma patient
}

\author{
Aude Quentin-Gondard ${ }^{1}$, Marielle Legoff ${ }^{2,3}$, Myriam Edjlali $^{2,4}$, Guillaume Geri ${ }^{2,5}$, Barbara Burroni2, ${ }^{2,6}$, Benedicte Deau ${ }^{2,3}$, Lise Willems ${ }^{2,3}$, \\ Dominique Magli-Barioz ${ }^{3}$, Farhad Heshmati ${ }^{3}$, Frédéric Pène ${ }^{2,5}$, David Calvet ${ }^{2,7}$, Didier Bouscary ${ }^{2,3}$ and Jerome Tamburini' ${ }^{2,3 *}$ \\ ${ }^{1}$ Centre Hospitalier Jean Jaurès, 75019 Paris, France \\ ${ }^{2}$ Université Paris Descartes, Faculté de Médecine Sorbonne Paris Cité, 75005 Paris, France \\ ${ }^{3}$ Hematology Department, Cochin Hospital, Assistance Publique-Hôpitaux de Paris (AP-HP), 75014 Paris, France \\ ${ }^{4}$ Service Imagerie Morphologique et Fonctionnelle, Centre Hospitalier Sainte Anne, 75014 Paris, France \\ ${ }^{5}$ Medical Intensive Care Unit, Cochin Hospital, Assistance Publique-Hôpitaux de Paris (AP-HP), 75014 Paris, France \\ ${ }^{6}$ Pathology Department, Cochin Hospital, Assistance Publique-Hôpitaux de Paris (AP-HP), 75014 Paris, France \\ ${ }^{7}$ Neurology Department, Centre Hospitalier Sainte Anne, 75014 Paris, France
}

\begin{abstract}
\section{Introduction}

While neurological and meningeal tumoral involvement is frequent in aggressive B-cell lymphoma-and a hallmark of Burkitt lymphomaelective spinal cord localization is uncommon. Here, we report the case of a 42-year-old female with stage IV Burkitt lymphoma who developed an early myelopathy on therapy with rapid progression.
\end{abstract}

We report a case of acute myelopathy in a patient with Burkitt lymphoma successfully treated by high-dose therapy (TBuCy) and autologous stem cell transplantation. Lymphoma involvement and methotrexate toxicity are discussed as potential causal mechanisms for neurological symptoms.

\section{Case report}

She presented with acute weight-loss and asthenia along with B-symptoms including night sweats and fever. She also had diffuse bone pain and a typical numb chin syndrome but her neurological examination was normal. She had anemia (hemoglobin $86 \mathrm{~g} / \mathrm{l}$, normal range $120-170 \mathrm{~g} / \mathrm{l}$ ) and thrombocytopenia (platelets $59 \times 10^{9} / \mathrm{l}$, normal range $150-450 \times 10^{9} / \mathrm{l}$ ), and bone marrow biopsy revealed massive involvement by monomorphic medium-sized B-cells with morphological and immunophenotypic characteristics of Burkitt lymphoma. Routine blood tests showed a more than 20 -fold increased lactate dehydrogenase (LDH) level (4878 UI/l, normal range 135-214 $\mathrm{UI} / \mathrm{l}$ ), moderate liver tests abnormalities (AST $146 \mathrm{UI} / \mathrm{l}$, normal range 10-45 UI/l; ALT 38, normal range 10-35 UI/l; GGT $381 \mathrm{UI} / 1$, normal range 7-35 UI/l; APL $232 \mathrm{UI} / 1$, normal range 35-120 UI/l and total bilirubin $16.9 \mu \mathrm{M}$, normal range 3-17 $\mu \mathrm{M}$ ) and a normal renal function (creatinine $38 \mu \mathrm{M}$, normal range $45-100 \mu \mathrm{M}$ ). Cerebrospinal fluid examination was normal (absence of visible cell; $0.33 \mathrm{~g} / \mathrm{l}$ protein and $2 \mathrm{mM}$ glycorachia with $3.6 \mathrm{mM}$ glycemia). Brain and spine magnetic resonance imaging (MRI) were normal. No significant lymphatic or systemic involvement was observed on PET/CT scan. Finally, she was diagnosed with stage IV Burkitt lymphoma with bone marrow and possible meningeal involvement. She received emergency chemotherapy with a COP regimen including $460 \mathrm{mg}$ cyclophosphamide day $1 ; 1.5 \mathrm{mg}$ vincristine day 1 and $60 \mathrm{mg}$ prednisone day 1 through 5 , along with three therapeutic lumbar punctures ( $15 \mathrm{mg}$ methotrexate, $40 \mathrm{mg}$ cytarabine and $20 \mathrm{mg}$ hydrocortisone) during the first six days of treatment. An improvement of chin dysesthesia was observed at this stage. She had an excellent response to COP as day 7 bone marrow examination showed less than $5 \%$ of lymphoma infiltration. Then she received vincristine $2 \mathrm{mg}$ day 1 , methotrexate $4300 \mathrm{mg}$ day 1 , doxorubicine $86 \mathrm{mg}$ day 2, cyclophosphamide $720 \mathrm{mg}$ days 2 through 4, prednisolone $90 \mathrm{mg}$ day 1 through 6 (the COPADM regimen), rituximab $600 \mathrm{mg}$ at days 6 and 14 and 3 additional intrathecal injections. At day 7 from the onset of COPADM, she experienced sudden lower limb deficiency, bladder dysfunction and T10-T11 sensitive level with a complete loss of proprioception, pain and temperature sensation, along with the recurrence of numb chin syndrome. Emergency spine MRI excluded a spinal compression or myelitis. A cerebrospinal fluid examination showed an increased protein level (1.6 g/l) without lymphoma cells. In the absence of other cause of paraplegia and sensory loss, and in a context of progressive numb chin syndrome, she was considered as having a neurological widespread of her disease and received additional high-dose methotrexate (11 $500 \mathrm{mg}$ ). A few days later, improvements of her legs and chin sensitivity was observed. Then she received CYVE (cytarabine $70 \mathrm{mg}$ over $12 \mathrm{~h}$ day 1 through 5; cytarabine $4300 \mathrm{mg}$ over 3 h day 2 through 5; etoposide $290 \mathrm{mg}$ day 2 through 5) followed by a stem cell collection upon G-CSF mobilization. Unfortunately, her neurological condition worsened during this treatment, with the occurrence of lower limbs hyperalgesia and a progression of her sensory loss to a T6 level. At this time, spine MRI showed features of conus medullaris hemorrhagic infarction and a C6-C7 intramedullary FLAIR hyperintensity suggesting a spinal cord ischemia (Figure 1A). She received a salvage therapy by CYM (methotrexate $4300 \mathrm{mg}$ day 1

Correspondence to: Jerome Tamburini, Service d'Hématologie Clinique, Hôpital Cochin, 27 rue du Faubourg Saint Jacques, 75014 Paris, France, Tel: 33158412121; Fax: 33184106322; E-mail: jerome.tamburini@aphp.fr

Key words: myelopathy, Burkitt lymphoma, methotrexate, autologous stem cell transplant

Received: May 22, 2017; Accepted: June 16, 2017; Published: June 20, 2017 
and cytarabine $145 \mathrm{mg}$ over $24 \mathrm{~h}$, day 2 through 6). Ten days after this therapy, she was referred to intensive care unit for mechanical ventilation due to the extension of her neurological defect to a T4 level with acute respiratory failure. After discussion with the patient, her family and the medical teams, conditioning regimen for autologous stem cell transplantation was given using thiotepa $360 \mathrm{mg}$ days 1-3; busulfan 38 $\mathrm{mg}$ for a total of 10 infusions during 3 days and cyclophosphamide 2820 $\mathrm{mg}$ days 7-8 (TBuCy regimen), and autologous stem cells were injected $48 \mathrm{~h}$ after chemotherapy completion. She had a partial hematopoietic recovery 8 days after stem cell transplant. She recovered a spontaneous ventilation allowing a withdrawn of mechanical ventilation 11 days after transplant. Follow-up spine MRI showed extensive T2 hyperintensities from C7 to the conus medullaris (Figure 1B), with spontaneous T1 hyperintensities suggesting hemorragic transformation of ischemic lesions (Figures 1C and 1D). Then, she underwent a 6 month-long inhospital neurological, psychological and social rehabilitation. She is now two years from transplant in persistent complete remission of her lymphoma, and lives at home with environment accommodations and daily nurse care.

\section{Discussion}

Sudden onset of neurological sign associated with normal initial MRI suggested spinal cord ischemia. Characteristics of one-month MRI signal were consistent with this mechanism, but the large extension of the spinal T2 hyperintensity - beyond the segmentation of spinal arterial vascularization - observed during follow-up is uncommon. Hence, we discussed a potential neurological toxicity of methotrexate, which hypothesis was reinforced by the detection of brain MRI FLAIR white matter hyperintensities that may have been cause by high-dose

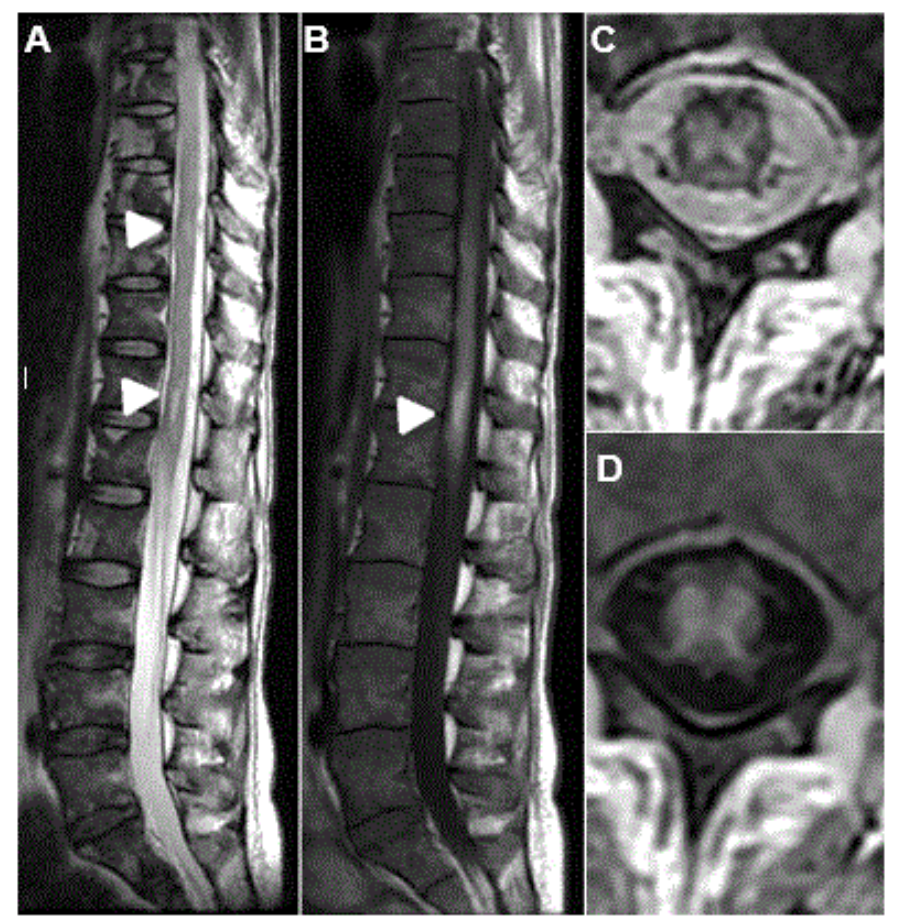

Figure 1. Follow-up MRI spine imaging. A) Extensive T2 hyperintense signals appeared onfollow-up (head arrows). Of note, the heterogeneity of the vertebrae, suggesting a lymphomatous bone infiltration. B) Extensive abnormalities of the spinal cord (from C7 to the conus medullary) with spontaneous $\mathrm{T} 1$ hyperintense signals centered on the conus medullary, without enhancement, suggesting hemorrhagic lesions. C) Two-dimension Axial T2 showed a global T2 hyperintense signal of the spinal cord's grey matter. D) Spontaneous $\mathrm{T} 1$ hyperintense signals suggesting hemmorhagic transformation of the ischemic lesion. methotrexate therapy. However, most cases of methotrexate-related myelopathies were reported with intrathecal administration [1-4], suggesting a local rather of systemic mechanism for these toxicities. The concomitance of progressive numb chin syndrome and the transient improvement of neurological symptoms upon high-dose methotrexate rather suggested that neurological symptoms were lymphoma-related. The absence of extrinsic or intrinsic tumor compression suggested intravascular lymphoma diffusion, while this topography is only reported in intravascular lymphoma (IVL), a rare diffuse large B-cell lymphoma variant [5]. We first used high-dose methotrexate and cytarabine, either combined or sequentially administrated for their diffusion into central nervous system [6]. However, the hypothesis of methotrexate CNS toxicity, even as a concomitant factor prompted us to change our therapeutic strategy. We thus performed a salvage autologous stem cell transplantation using a blood-brain barrier disrupting conditioning chemotherapy. The TBuCy regimen is a widely approved regimen for CNS lymphoma, allowing long-term disease control even in diseases refractory to chemotherapy [7]. Moreover, this regimen is associated with acute toxicities (particularly neutropenic fever and infection) responsible of a 5-7\% toxicity-related mortality, but is not associated with acute or late neurological toxicities [8,9]. In our case, we observed a rapid neurological response after autologous transplant, as attested by mechanical ventilation withdrawn and gradual improvement of motor functions, suggesting that her neurological symptoms were mostly lymphoma-related.

\section{Conclusion}

With a two-year post-transplantation follow-up, our patient is still in complete remission, and had a slow but progressive improvement of her autonomy. She and her family now consider they have a good quality of life despite her persistent disability. Our current report supports the use of repeated central nervous system imaging in case of neurological symptoms in lymphoma patients. Because distinguishing spine toxicity of methotrexate from disease-specific involvement is challenging, we suggest considering autologous stem cell transplantation using nonneurotoxic blood-brain barrier disrupting conditioning chemotherapy as frontline treatment in lymphoma patients with progressive neurological symptoms.

\section{References}

1. Cachia D, Kamiya-Matsuoka C, Pinnix CC, Chi L, Kantarjian HM, et al. (2015) Myelopathy following intrathecal chemotherapy in adults: a single institution experience. J Neurooncol 122: 391-398. [Crossref]

2. Counsel P, Khangure M (2007) Myelopathy due to intrathecal chemotherapy: magnetic resonance imaging findings. Clin Radiol 62: 172-176. [Crossref]

3. Joseph PJ, Reyes MR (2014) Dorsal column myelopathy following intrathecal chemotherapy for acute lymphoblastic leukemia. J Spinal Cord Med 37: 107-113. [Crossref]

4. Murata KY, Maeba A, Yamanegi M, Nakanishi I, Ito H (2015) Methotrexate myelopathy after intrathecal chemotherapy: a case report. J Med Case Rep 9: 135. [Crossref]

5. Tahsili-Fahadan P, Rashidi A, Cimino PJ, Bucelli RC, Keyrouz SG (2016) Neurologic manifestations of intravascular large B-cell lymphoma. Neurol Clin Pract 6: 55-60. [Crossref]

6. Ferreri AJ (2011) How I treat primary CNS lymphoma. Blood 118: 510-522. [Crossref]

7. Soussain C, Hoang-Xuan K, Taillandier L, Fourme E, Choquet S, et al. (2008) Intensive chemotherapy followed by hematopoietic stem-cell rescue for refractory and recurrent primary CNS and intraocular lymphoma: Societe Francaise de Greffe de Moelle Osseuse-Therapie Cellulaire. J Clin Oncol 26: 2512-2518. [Crossref] 
8. Scordo M, Bhatt V, Hsu M, Omuro AM, Matasar MJ, et al. (2017) A Comprehensive Assessment of Toxicities in Patients with Central Nervous System Lymphoma Undergoing Autologous Stem Cell Transplantation Using Thiotepa, Busulfan, and Cyclophosphamide Conditioning. Biol Blood Marrow Transplant 23: 38-43.
9. Soussain C, Choquet S, Fourme E, Delgadillo D, Bouabdallah K, et al. (2012) Intensive chemotherapy with thiotepa, busulfan and cyclophosphamide and hematopoietic stem cell rescue in relapsed or refractory primary central nervous system lymphoma and intraocular lymphoma: a retrospective study of 79 cases. Haematologica 97: 17511756. [Crossref]

Copyright: (C2017 Quentin-Gondard A. This is an open-access article distributed under the terms of the Creative Commons Attribution License, which permits unrestricted use, distribution, and reproduction in any medium, provided the original author and source are credited. 